\title{
Comparando as opiniões do professor e seus alunos sobre o uso de um laboratório virtual de robótica: um relato de experiência
}

\author{
Victor Vieira' $^{1,2}$, Albert Costa ${ }^{1}$, Bruno Gadelha ${ }^{1}$ \\ ${ }^{1}$ Instituto de Computação - Universidade Federal do Amazonas (UFAM) \\ 69.077-000 - Manaus - AM - Brasil
}

${ }^{2}$ Grupo de Informática para Pesquisa em Computação - Instituto Federal do Acre (IFAC)

69.903-068 - Acre - AC - Brasil

victor.vieira@ifac.edu.br, albertjosua@yahoo.com.br,

bruno@icomp.ufam.edu.br

\begin{abstract}
This work presents an experience report with the use of a virtual laboratory for teaching robotics. High school students from the Informatic course of the Instituto Federal do Amazonas participated in an extension course in robotics and developed activities in the Arduino simulator SimulIDE. Teacher and students opinions on the activities were collected and analyzed in the article. The results point to the virtual laboratory used as a useful tool in the preparation for the development of activities in the real laboratory and as a motivating factor.
\end{abstract}

Resumo. Este trabalho apresenta um relato de experiência com a utilização de um laboratório virtual para o ensino de robótica. Alunos do ensino médio do curso Técnico em Informática do Instituto Federal do Amazonas participaram de um curso de extensão em robótica e desenvolveram atividades no simulador de Arduino SimulIDE. As opiniões do professor e dos alunos sobre as atividades foram coletadas e são analisadas no artigo. Os resultados apontam para o laboratório virtual utilizado como uma ferramenta útil na preparação para o desenvolvimento das atividades em laboratório real e como um fator motivador.

\section{Introdução}

Com o surgimento de novas Tecnologias Digitais de Informação e Comunicação (TDIC), a educação vem se adaptando aos dispositivos e ferramentas que surgem para serem utilizados no ensino. Os professores possuem diversas possibilidades de tornar o ambiente da sala de aula mais interativo e motivar os alunos a aprenderem de diferentes maneiras. Uma dessas possibilidades são os Softwares Educativos (SEs) [Godoi e Padovani, 2009].

Os SEs são programas de computador desenvolvidos para ensinar. Uma das categorias de SEs existentes é a dos Laboratórios Virtuais (LVs), onde os alunos podem realizar experimentos ou simular atividades como ocorrem nos laboratórios reais [Koh, 2017]. Como vantagens, essas atividades geralmente são simuladas em um ambiente seguro (laboratório de informática) e com custos menores [Diwakar et al., 2016; Melo e 
VII Congresso Brasileiro de Informática na Educação (CBIE 2018)

Anais do XXIV Workshop de Informática na Escola (WIE 2018)

Osso Jr., 2008]. Além disso, o planejamento e execução de uma atividade num Laboratório Virtual (LV) tende a ser mais fácil, pois a preparação do ambiente costuma ser mais simples [Mikhaylov e Chernov, 2012].

Neste trabalho, é relatada uma experiência com a utilização do LV SimulIDE para o ensino de robótica num curso de extensão de curta duração para alunos do ensino médio do curso Técnico em Informática do Campus Humaitá do Instituto Federal do Amazonas (IFAM). As opiniões do professor e dos alunos sobre as atividades desenvolvidas no LV foram registradas através de questionários específicos e são comparadas.

Alguns trabalhos, como os de Guillermo [2017], Bordini et al. [2017], Benitti e Fiori [2010] e Falcão e Machado [2010] tratam do uso de LVs em atividades de ensino. Porém, os objetivos e resultados são diferentes dos deste trabalho. Guillermo [2017] apresenta uma metodologia de uso de um LV de Engenharia baseada em resultados positivos, quando utilizada. Bordini et al. [2017], Benitti e Fiori [2010] e Falcão e Machado [2010] apresentam ferramentas que podem ser utilizadas no ensino de Química e Matemática, respectivamente, mas não investigam sua utilização à fundo. Nenhum dos trabalhos encontrados compara os pontos de vista do professor e seus alunos com relação ao uso de um LV.

O restante do texto está organizado da seguinte maneira: na Seção 2, são descritos os métodos executados, explicando todas as etapas do trabalho; na Seção 3, são descritos os resultados e discussão das opiniões do professor e alunos sobre o uso do LV; e na Seção 4, são descritas as conclusões, com os objetivos alcançados e propostas de trabalhos futuros.

\section{Métodos}

Este relato de experiência ocorreu de acordo com os procedimentos ilustrados na Figura 1, contendo as seguintes etapas: planejamento e execução do curso; registro das experiências de uso do LV; e análise dos resultados.

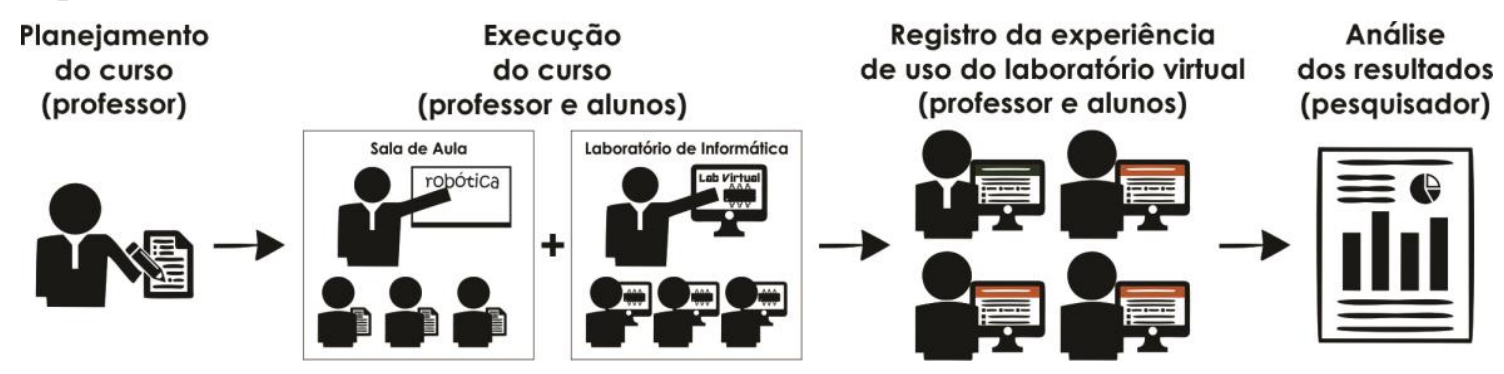

Figura 1. Etapas desenvolvidas

Inicialmente, foi planejado um curso de extensão de robótica com duração de 4 horas para alunos do curso Técnico Integrado ao Ensino Médio em Informática do Campus Humaitá do IFAM. A ementa do curso contemplou conceitos básicos sobre robótica e programação de microcontroladores Arduino. A carga horária foi dividida em 2 horas teóricas em sala de aula, com a introdução de conceitos relacionados a robótica e programação para Arduino, e 2 horas práticas no laboratório de informática, para o desenvolvimento das atividades no LV. A Figura 2 mostra a realização das atividades no laboratório de informática da instituição. 
VII Congresso Brasileiro de Informática na Educação (CBIE 2018)

Anais do XXIV Workshop de Informática na Escola (WIE 2018)
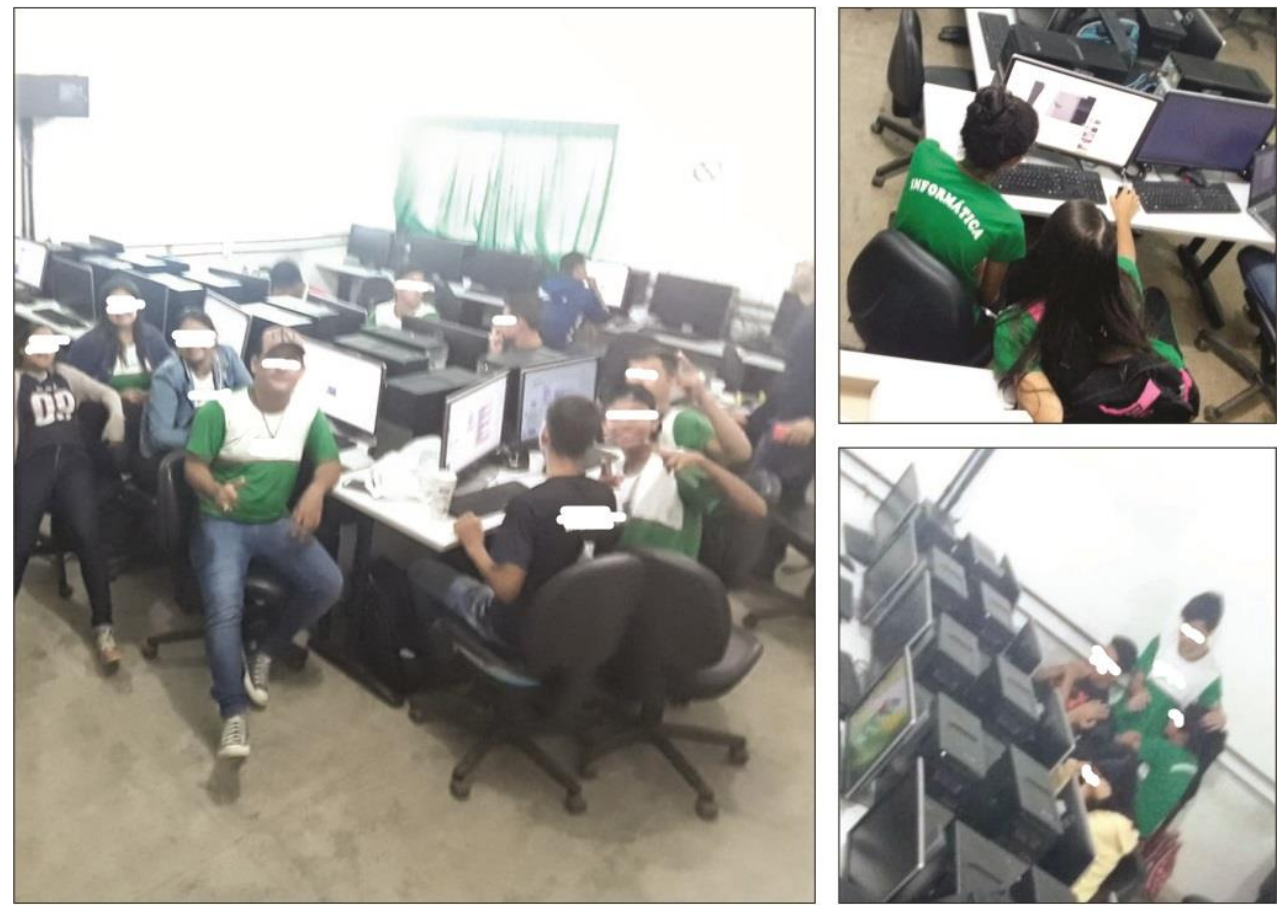

Figura 2. Atividades do curso de extensão de robótica no Laboratório de Informática do IFAM

O laboratório virtual utilizado para a prática foi o SimulIDE, escolhido pelo professor, Mestre em Informática com experiência em robótica, por ser um simulador de circuito eletrônico em tempo real de interface simples que fornece um microcontrolador Arduino. A interface do SimulIDE pode ser vista na Figura 3. Vinte e um (21) alunos frequentaram o curso, que ocorreu em um (1) dia.

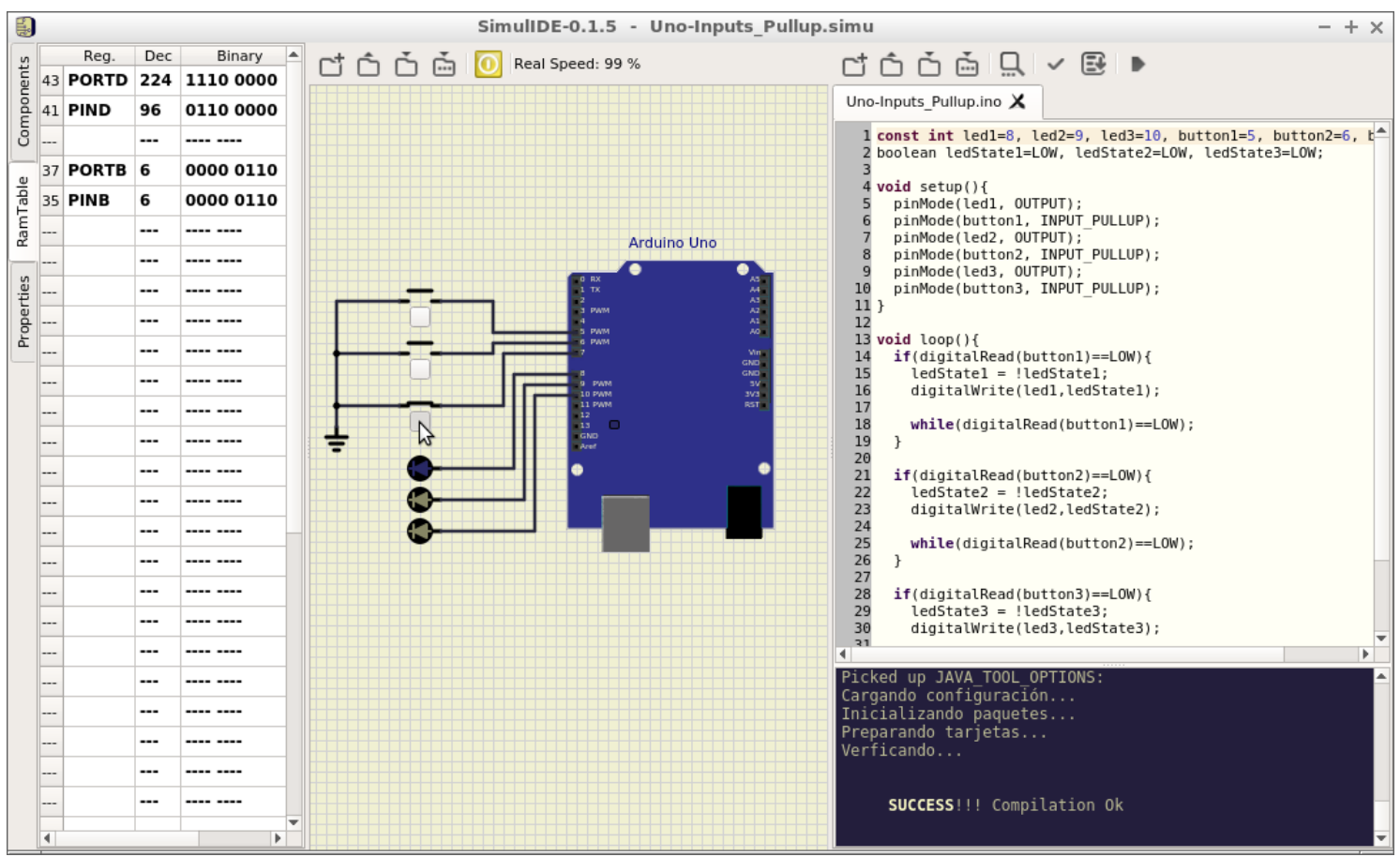

Figura 3. Interface do SimulIDE 
VII Congresso Brasileiro de Informática na Educação (CBIE 2018)

Anais do XXIV Workshop de Informática na Escola (WIE 2018)

As experiências no uso do LV foram registradas ao final do curso. Para registrar a experiência do professor, foi utilizado o Instrumento para Registro da Experiência Docente no Uso de Laboratórios Virtuais, disponível no Relatório Técnico RT-GSI2018-004 [Vieira e Gadelha, 2018]. Consiste em um questionário que aborda, do ponto de vista docente, o contexto de uso, a metodologia utilizada, o suporte fornecido aos alunos, a facilidade de uso percebida através das dificuldades dos alunos, a motivação percebida nos alunos, a fidelidade da simulação, as características do acesso ao LV, o desempenho dos alunos e observações adicionais. As informações obtidas com o instrumento refletem como ocorreu uma atividade com um LV na opinião do professor.

A partir desse instrumento, foi desenvolvido um questionário para registrar o ponto de vista dos alunos com relação às atividades com o LV. No desenvolvimento deste questionário, buscou-se abordar tópicos que possibilitassem a comparação dos resultados com os do instrumento para o professor. $\mathrm{O}$ questionário, que pode ser visto na Tabela 1, é anônimo, foi disponibilizado como Formulário do Google e contém 10 questões, entre em escala Likert de cinco pontos e abertas.

Tabela 1. Questionário utilizado para registrar as opiniões dos alunos sobre a atividade com o laboratório virtual

\begin{tabular}{|c|c|c|}
\hline & Tipo & Questões \\
\hline 1 a & Likert & Achei fácil utilizar o laboratório virtual. \\
\hline 2 a & Likert & Me senti motivado a resolver os problemas durante toda a atividade. \\
\hline 3a & Likert & Recebi ajuda do professor para concluir a atividade. \\
\hline $4 \underline{a}$ & Likert & Recebi ajuda de um colega para concluir a atividade. \\
\hline 5 & Likert & Ao término da atividade, me senti preparado para realizá-la em um ambiente real. \\
\hline 6a & Likert & O tempo destinado pelo professor foi suficiente para concluir a atividade. \\
\hline 7ạ & Aberta & O que achou da metodologia utilizada pelo professor para a atividade? \\
\hline 8 a & Aberta & Quais os pontos positivos da atividade realizada? \\
\hline 9ạ & Aberta & Quais os pontos negativos da atividade realizada? \\
\hline $10^{\mathrm{a}}$ & Aberta & Descreva as observações adicionais. \\
\hline
\end{tabular}

Os resultados foram analisados individualmente, em cada perspectiva (professor e alunos), e comparados, ao final. As respostas do professor foram analisadas de forma qualitativa de maneira discursiva, pois constituem uma única amostra. Já as respostas dos alunos foram analisadas quantitativa e qualitativamente: as questões em escala Likert tiveram análise quantitativa; na questão sobre metodologia, a análise foi qualitativa, discutindo sobre a opinião dos alunos em relação a como o professor conduziu a atividade; nas demais questões abertas, utilizou-se o Método de Inspeção do Discurso Subjascente (MEDS) [Nicolaci-Da-Costa, 2007], baseado na classificação das opiniões em categorias, para análise qualitativa. $\mathrm{Na}$ comparação, os resultados foram classificados pelos itens facilidade de uso, motivação, suporte, preparação e metodologia, que são abordados nos dois questionários e foram considerados importantes para esse relato de experiência.

\section{Resultados e Discussão}

Os resultados e discussão deste relato de experiência são apresentados a seguir e surgem a partir: a) das respostas do professor ao Instrumento para Registro da Experiência Docente no uso de Laboratórios Virtuais (Subseção 3.1); b) das respostas dos alunos ao questionário desenvolvido para registrar seu ponto de vista com relação às atividades 
VII Congresso Brasileiro de Informática na Educação (CBIE 2018)

Anais do XXIV Workshop de Informática na Escola (WIE 2018)

com o LV (Subseção 3.2); e c) da comparação das respostas do professor e dos alunos (Subseção 3.3).

\subsection{Análise do ponto de vista do professor sobre a atividade}

Os resultados do registro da experiência de uso do LV realizado pelo professor descrevem o contexto do uso como o apresentado na Seção 2: um curso de extensão de robótica com 4 horas de carga horária para 21 alunos de ensino médio técnico, sendo 2 horas utilizando o laboratório virtual SimulIDE para simular um microcontrolador Arduino.

Com relação à metodologia, o professor propôs uma atividade básica, para que os alunos conseguissem fazer com que uma luz de LED acendesse no Arduino. O acesso foi individual para que a avaliação do desempenho pudesse ser também individualizada. O simulador foi acessado através de computadores por serem o dispositivo existente no laboratório de informática da instituição e não ter sido encontrada versão do software para outros dispositivos. Destaca-se que o simulador foi considerado difícil de ser instalado pelo professor.

Com relação ao suporte fornecido aos alunos, o professor afirmou ter instruído antes do uso sobre o SimulIDE e sobre o conteúdo abordado nele e, ainda, durante o uso apenas com relação ao funcionamento do LV. Os alunos não solicitaram auxílio com relação aos conceitos que estavam sendo desenvolvidos no simulador enquanto $o$ utilizavam. O professor ainda afirmou ter percebido problemas e dificuldades no uso por parte dos alunos e considerou que os mesmos estiveram desmotivados.

A fidelidade do LV foi classificada como alta, tendo sido considerado que ele simula as atividades propostas exatamente como ocorrem em ambiente real e que os alunos saíram preparados para executar os mesmos procedimentos em um laboratório real. O desempenho geral foi classificado como "bom", numa escala de "péssimo" a "excelente".

Analisando as respostas do professor ao Instrumento para Registro da Experiência Docente no Uso de Laboratórios Virtuais, percebe-se que as atividades foram bem planejadas e, mesmo assim, na opinião dele, os alunos não estiveram motivados e houve dificuldades e problemas. Entretanto, isso não prejudicou a preparação para o desenvolvimento das atividades no laboratório real nem o desempenho dos alunos, que foi positivo.

\subsection{Análise do ponto de vista dos alunos sobre a atividade}

Os 21 alunos que participaram do curso responderam a seis questões em escala Likert de cinco pontos - de "discordo totalmente" (1) a "concordo totalmente" (5) - e quatro questões abertas. A Tabela 2, a seguir, apresenta os resultados em média e desvio padrão das questões em escala Likert, permitindo a visualização da opinião geral dos alunos nesses tópicos e se houve grande discordância entre as respostas. $\mathrm{O}$ desvio padrão foi considerado elevado a partir de 1,40, para a quantidade de alunos e escala utilizada. 
VII Congresso Brasileiro de Informática na Educação (CBIE 2018)

Anais do XXIV Workshop de Informática na Escola (WIE 2018)

Tabela 2. Resultados das questões em escala Likert para registro das opiniões dos alunos sobre a atividade com o LV

\begin{tabular}{llcc}
\cline { 2 - 4 } & Questão & Média & Desvio padrão \\
\hline 1ạ $^{\text {a }}$ & Achei fácil utilizar o laboratório virtual. & $\mathbf{3 , 7 6}$ & $\mathbf{1 , 1 3}$ \\
\hline $2^{\text {a }}$ & Me senti motivado a resolver os problemas durante toda a atividade. & $\mathbf{3 , 6 6}$ & $\mathbf{1 , 3 1}$ \\
\hline $3^{a \underline{a}}$ & Recebi ajuda do professor para concluir a atividade. & $\mathbf{2 , 0 4}$ & $\mathbf{1 , 5 9}$ \\
\hline $4^{\underline{a}}$ & Recebi ajuda de um colega para concluir a atividade. & $\mathbf{1 , 9 0}$ & $\mathbf{1 , 5 4}$ \\
\hline $5^{\underline{a}}$ & Ao término da atividade, me senti preparado para realizá-la em um ambiente real. & $\mathbf{3 , 5 2}$ & $\mathbf{1 , 4 0}$ \\
\hline $6^{\underline{a}}$ & O tempo destinado pelo professor foi suficiente para concluir a atividade. & $\mathbf{3 , 8 0}$ & $\mathbf{1 , 5 0}$ \\
\hline
\end{tabular}

Considerando a escala Likert adotada, de "discordo totalmente" (1) a "concordo totalmente" (5), percebe-se que:

- A maioria dos alunos julgou ser fácil utilizar o LV (67\% concordaram com a afirmação da primeira questão e a média foi próxima de 4,0 );

- A maioria dos alunos se sentiu motivada durante a atividade (62\% concordaram com a afirmação da segunda questão e a média foi acima de $3,0)$;

- A minoria dos alunos foi ajudada pelo professor ou por um colega para concluir a atividade (67\% discordaram da afirmação da terceira questão, $71 \%$ discordaram da afirmação da quarta questão e as médias foram próximas de 2,0);

- A maioria dos alunos se sentiu preparada para realizar a atividade desenvolvida num laboratório real $(62 \%$ concordaram com a afirmação da quinta questão e a média foi acima de 3,0$)$;

- A maioria dos alunos concluiu a atividade proposta no tempo disponibilizado pelo professor $(76 \%$ concordaram com a afirmação da sexta questão e a média foi próxima de 4,0);

- O baixo desvio padrão na $1^{\mathrm{a}}, 2^{\mathrm{a}}$ e $5^{\mathrm{a}}$ questão indica que os alunos concordaram entre si nas respostas com relação à facilidade de uso, motivação e preparação para a prática em ambiente real;

- O alto desvio padrão na $3^{\mathrm{a}}, 4^{\mathrm{a}}$ e $6^{\mathrm{a}}$ questão indica que houve grande distanciamento, ou seja, discordância, entre as respostas dos alunos com relação ao auxílio recebido do professor ou de um colega e tempo destinado para o desenvolvimento da atividade.

Perguntados sobre o que acharam da metodologia utilizada pelo professor, a maioria dos alunos considerou ter sido pouco explicativa, com comentários como " $O$ professor explicou a atividade mas pouca gente entendeu, porque não estudamos Arduino no IFAM" e "achei que poderia ter mais explicação”. Alguns, nessa questão, acabaram avaliando a atividade realizada no LV como "dificil". Houve apenas seis comentários positivos sobre a metodologia utilizada, no sentido de que "a metodologia foi boa".

Os pontos positivos e negativos e as observações adicionais eram campos não obrigatórios do questionário e foram analisados qualitativamente com o MEDS. As categorias que emergiram dessa análise foram as seguintes, com a quantidade de 
VII Congresso Brasileiro de Informática na Educação (CBIE 2018)

Anais do XXIV Workshop de Informática na Escola (WIE 2018)

ocorrências entre parênteses: novidade (14), metodologia (6), facilidade de uso (2) e simulação (1). Os comentários da categoria novidade estiveram mais relacionados a problemas, principalmente ao fato de alguns alunos nunca terem tido contato com robótica. Eles escreveram no seguinte sentido: "Arduino é dificil, não temos essa disciplina"; "não conheço Arduino" e "não estudamos robótica no IFAM". Na categoria metodologia, a maioria dos comentários foi relacionada ao fato de ter havido pouca explicação, na opinião dos alunos. Os comentários das categorias facilidade de uso e simulação são positivos, reforçando que acharam "fácil usar o LV" e "permite simular Arduino sem perigo de estragar”.

\subsection{Comparação entre os pontos de vista}

Os resultados dos registros da experiência de uso do SimulIDE dos pontos de vista do professor e dos alunos foram comparados com relação a cinco tópicos: facilidade de uso, motivação, suporte, preparação e metodologia. Todos esses tópicos são abordados nos dois questionários e foram considerados importantes para esse relato de experiência. A Tabela 3 apresenta a comparação.

Tabela 3. Comparação entre o ponto de vista do professor e o dos alunos

\begin{tabular}{|c|c|c|c|}
\hline Tópicos & Ponto de vista do professor & & Ponto de vista dos alunos \\
\hline Facilidade de Uso & $\begin{array}{r}\text { Os alunos tiveram dificuldades e } \\
\text { encontraram problemas }\end{array}$ & $\neq$ & Foi fácil utilizar o LV \\
\hline Motivação & $\begin{array}{r}\text { Os alunos não estiveram } \\
\text { motivados }\end{array}$ & $\neq$ & Estivemos motivados \\
\hline Suporte & $\begin{array}{r}\text { Foi fornecido suporte antes e } \\
\text { durante a atividade }\end{array}$ & $\neq$ & $\begin{array}{l}\text { Não fomos ajudados durante a } \\
\text { atividade }\end{array}$ \\
\hline Preparação & $\begin{array}{r}\text { Ao final da atividade, os alunos } \\
\text { estão preparados para a prática } \\
\text { no laboratório real }\end{array}$ & $=$ & $\begin{array}{l}\text { Ao final da atividade, estamos } \\
\text { preparados para a prática no } \\
\text { laboratório real }\end{array}$ \\
\hline Metodologia & $\begin{array}{r}\text { Bem estabelecida, com } \\
\text { planejamento e atividades básicas }\end{array}$ & $\neq$ & $\begin{array}{l}\text { Pouca explicação e atividade } \\
\text { difícil }\end{array}$ \\
\hline
\end{tabular}

Percebe-se que as opiniões divergem em quatro dos cinco tópicos. Com relação à facilidade de uso, apesar de os alunos terem considerado fácil utilizar o LV, o professor relatou dificuldades e problemas durante o uso. Destaca-se que, para o professor, o instrumento de registro da opinião não abordava diretamente a facilidade de uso do software para os alunos, mas sim o fato de terem encontrado dificuldades e problemas. Essa facilidade de uso pode ter sido interpretada pelos alunos como quão fácil é a interface do SimulIDE, enquanto para o professor, possivelmente foi interpretada como quão fácil foi concluir a atividade proposta na ferramenta. Seria necessária uma investigação aprofundada para determinar um consenso sobre esse tópico.

Com relação à motivação, percebe-se que, enquanto os alunos se sentiram motivados, o professor não foi capaz de percebê-la e julgou o contrário. Uma das causas da não percepção por parte do professor da real motivação dos alunos em realizar a atividade pode ser o fato de ter analisado apenas alguns aspectos relacionados ao comportamento [Neves e Boruchovitch, 2007]. Porém, o ideal seria a utilização de um procedimento bem estabelecido para essa avaliação. 
Com relação ao suporte, as opiniões também divergem, com o professor relatando que forneceu suporte antes e durante a atividade e os alunos afirmando que não foram ajudados. Nesse tópico, entretanto, destaca-se que a questão aos alunos não verificava se eles solicitaram ajuda e não receberam, mas se eles foram auxiliados. Pode ter ocorrido de não julgarem necessário o auxílio do professor, que esteve disponível. Ao mesmo tempo, o relato do professor sobre as dificuldades encontradas pelos alunos leva a concluir que eles deveriam ter solicitado ajuda e não o fizeram, mas não é possível afirmar os motivos para isso.

Com relação à preparação, há um consenso no sentido de que os alunos estão preparados para realizar a atividade que simularam em um laboratório real. Isso indica que o SimulIDE é uma ferramenta útil ao processo de ensino de microcontroladores Arduino quando a intenção é a capacitação sobre os procedimentos e para o uso dos equipamentos reais.

Com relação à metodologia, novamente as opiniões são contrárias. Apesar de o professor ter descrito todos os procedimentos realizados, tendo esses sido considerados bem planejados, e ter classificado a atividade desenvolvida como básica, os alunos opinaram necessitar de mais explicações sobre o conteúdo, com críticas à metodologia utilizada pelo professor, e consideraram ter sido difícil desenvolver a atividade proposta. Após uma análise comparando as críticas à metodologia com outras respostas individuais, percebeu-se que esses tendem a serem os alunos que tiveram mais dificuldades durante a utilização do LV, com as menores notas para facilidade de uso e motivação, no geral. Logo, cabe ao professor levar em considerações as opiniões para novas experiências, mas o fato dessas opiniões não serem em sua maioria positivas não conclui que a metodologia utilizada foi inadequada.

Como resultado geral dessa comparação, tem-se que: a) o LV foi fácil de ser usado pelos alunos, que estiveram motivados, apesar das dificuldades percebidas pelo professor; b) os alunos, mesmo com dificuldades, não solicitaram ajuda do professor, que esteve disponível e atendeu a alguns; c) o SimulIDE preparou os alunos para a prática com microcontroladores Arduino reais; e d) a metodologia utilizada pelo professor foi válida, mesmo com alguns alunos com menor desempenho criticando-a, mas pode ser revista para novas atividades.

\section{Conclusões}

Este trabalho relata uma experiência com o uso do laboratório virtual SimulIDE em um curso de extensão de robótica no IFAM. Os pontos de vista do professor e dos alunos sobre a atividade desenvolvida no SimulIDE foram coletados através de questionários específicos para o registro da experiência de uso do LV. Os dados foram analisados individualmente e comparados.

Percebeu-se que a utilização do LV foi interpretada de maneiras distintas pelo professor e seus alunos com relação à facilidade de uso, motivação, suporte e metodologia. Ambos concordaram totalmente apenas com o fato de que os alunos, após concluir a atividade, estão preparados para realizá-la no laboratório real. O SimulIDE se mostrou como uma ferramenta útil para a preparação para a prática em ambiente real e capaz de motivar os alunos. 
Entre as contribuições deste relato de experiência, estão seus resultados apontando para a utilização de LVs como fator motivador e capaz de preparar os alunos em contextos como o descrito no trabalho, além do questionário para registro da experiência dos alunos no uso do LV, que pode ser utilizado em outras situações em que se queira obter o ponto de vista discente sobre uma experiência com esse tipo de software. Como trabalhos futuros, espera-se: a) registrar outras experiências de uso de LVs com o questionário para alunos, solicitando opiniões sobre ele como forma de validá-lo; b) comparar os resultados de diferentes experiências de uso de LVs em amostras maiores; e c) utilizar o mesmo protocolo deste trabalho para realizar experiências com outros professores e turmas usando o SimulIDE e comparar os resultados com os deste estudo.

\section{Referências}

Benitti, F. e Fiori, T.. Supermercado Virtual: software educacional de matemática para o Ensino Fundamental. Anais do Workshop de Informática na Escola. 2010.

Bordini, R.; Otsuka, J.; Beder, D.; Silva, T.; Oliveira, L.; Camargo, A.; Ohnuma, C.; e Ribeiro, M.. LabTecA - Laboratório Virtual de Química Analítica. Anais do VI Congresso Brasileiro de Informática na Educação. 2017.

Diwakar, S.; Radhamani, R.; Sasidharakurup, H.; Kumar, D.; Nizar, N.; Achuthan, K.; e Nair, B.. Assessing Students and Teachers Experience on Simulation and Remote Biotechnology VL: A Case Study with a Light Microscopy Experiment. 2016.

Falcão, E. e Machado, L.. Um Laboratório Virtual Tridimensional e Interativo para Suporte ao Ensino de Física. Anais do Workshop de Informática na Escola. 2010.

Godoi, K. e Padovani, S.. Avaliação de material didático digital centrada no usuário: uma investigação de instrumentos passíveis de utilização. Produção, vol. 19, n. 3, 2009.

Guillermo, O.. Uso de laboratórios virtuais de aprendizagem em mecânica dos fluidos em Hidráulica na Engenharia. Anais do VI Congresso Brasileiro de Informática na Educação. 2017.

Koh, J.. Designing and integrating reusable learning objects for meaningful learning: Cases from a graduate programme. Australasian Journal of Educational Technology, n. 33, pg. 136-151, 2017.

Melo, R. e Osso Jr., J.. Laboratórios virtuais e ambientes colaborativos virtuais de ensino e de aprendizagem: conceitos e exemplos. Revista de Informática Aplicada, vol. IV, n. 02, 2008.

Mikhaylov, N. e Chernov, D.. From Virtual Lab to Virtual Development Lab. Proceedings of the 9th IFAC Symposium Advances in Control Education. Nizhny Novgorod, Russia, 2012.

Neves, E. e Boruchovitch, E.. Escala de avaliação da motivação para aprender de alunos do ensino fundamental (EMA). Psicol. Reflex. Crit., vol. 20, n. 3, pg. 406-413, 2007. 
VII Congresso Brasileiro de Informática na Educação (CBIE 2018)

Anais do XXIV Workshop de Informática na Escola (WIE 2018)

Nicolaci-Da-Costa, A. M.. O campo da pesquisa qualitativa e o Método de Explicitação do Discurso Subjacente (MEDS). Psicol. Ref. Crit., Porto Alegre, vol. 20, n. 1, , 2007.

Vieira, V. e Gadelha, B.. RT-GSI-2018-0004. Instrumento para Registro de Experiência Docente no Uso de Laboratórios Virtuais - Terceira Versão. Relatório Técnico do Grupo de Sistemas Inteligentes. 2018. Disponível em https://goo.gl/qVZv7b. 